\title{
Safety and immunogenicity of a SARS-CoV-2 inactivated vaccine in patients with chronic hepatitis $B$ virus infection
}

\author{
Tiandan Xiang ${ }^{1,2,3}$, Boyun Liang ${ }^{1,2,3}$, Hua Wang ${ }^{1,2,3}$, Xufeng Quan ${ }^{1,2}$, Shengsong He $\mathrm{H}^{1,2}$, Helong Zhou ${ }^{1,2}$, Yongwen He ${ }^{1,2}$, \\ Dongliang Yang ${ }^{1,2}$, Baoju Wang ${ }^{1,2}$ and Xin Zheng ${ }^{1,2 \bowtie}$
}

(c) The Author(s) 2021

Cellular \& Molecular Immunology (2021) 18:2679-2681; https://doi.org/10.1038/s41423-021-00795-5

Coronavirus disease 2019 (COVID-19), caused by SARS-CoV-2 infection, has become a major global public health threat. Although significant advances have been made in developing and applying different vaccines in clinical trials [1, 2], data are limited on the safety and efficacy of the inactivated vaccine in patients with chronic liver disease [3]. Recent studies have preliminarily described the safety and immunogenicity of SARSCoV-2 vaccines in patients with nonalcoholic fatty liver disease and in liver transplant recipients $[4,5]$. However, to date, there is no detailed information on the SARS-CoV-2 inactivated vaccine in patients with chronic hepatitis $B(\mathrm{CHB})$ infection. It has been reported that $\mathrm{CHB}$ patients have impaired immune systems [6]. Hence, whether immunocompromised CHB patients within the different clinical stages can be safely vaccinated with the various types of SARS-CoV- 2 vaccines and produce an effective immune response remains unclear. Our study aims to provide a comprehensive analysis from different clinical dimensions to characterize the safety and immunogenicity of SARS-CoV-2 inactivated vaccines (BBIBP-CorV, CoronaVac, or WIBP-CorV) within this specific patient population.

A total of $284 \mathrm{CHB}$ patients who were unvaccinated $(n=81)$ or had completed the first $(n=54)$ or second dose $(n=149)$ of the vaccines were enrolled from March 23, 2021, to September 10, 2021 (Table S1). The median time post-vaccination was 33 (IQR, 24-48) days among the 149 completely vaccinated patients. Safety was evaluated by determined the overall incidence of adverse reactions via a standardized questionnaire. Moreover, plasma samples were examined for IgG antibodies against the receptor-binding domain (RBD) of the SARS-CoV-2 spike protein (anti-S-RBD-lgG) and for neutralizing antibodies (NAbs). The complete methods regarding the study design and the statistical analysis are available in the Supplementary methods section.

The adverse reaction data were first analyzed in 149 completely vaccinated $\mathrm{CHB}$ patients. The overall incidence of adverse reactions within 7 days was $30.2 \%$ (Table S2), which was similar to that found in the phase 3 trials of CoronaVac in Turkey [2]. The most common side effect was injection-site pain $(25.5 \%, 38 / 149)$, followed by drowsiness (3\%,3/149); only one patient reported fever on the first day after vaccination. Almost all of the adverse reactions were mild and self-resolved within a few days after vaccination. Serious side effects were not observed even in
$20 \mathrm{CHB}$ patients with abnormal alanine aminotransferase levels [61.5 (43-129) U/L] or 10 patients with compensated liver cirrhosis. The results demonstrated that SARS-CoV-2 inactivated vaccines had a favorable safety profile in CHB patients. Given that previous studies have shown an increased risk of progression to severe disease in COVID-19 patients with cirrhosis [7], the benefit of vaccination in compensated cirrhotic patients still outweighs the vaccine-related risk.

Next, we determined the immunogenicity of CHB patients who completed the two doses of the vaccination regimen. The seropositivity for anti-S-RBD-IgG and NAbs was $87.25 \%$ and $74.5 \%$, respectively (Fig. $1 \mathrm{~A}$ ). The anti-S-RBD-IgG seropositivity of $\mathrm{CHB}$ vaccine recipients was similar to that in a clinical trial of CoronaVac in Turkey (89.7\%) but much higher than the reported recently seropositivity of IgG antibodies to the spike protein (76\%) in patients with chronic liver disease [5]. Both anti-S-RBD-IgG and $\mathrm{NAb}$ levels increased significantly to a higher level after completing the vaccination regimen (Fig. $1 B, C, P<0.0001$ ). This finding indicates that SARS-CoV-2 inactivated vaccines can elicit an optimal antibody response even though some $\mathrm{CHB}$ patients may have pre-existing compromised immune function.

The seropositivity and antibody titers in CHB patients were further compared according to sex, age, antiviral therapy, and body mass index stratification (Fig. 1D, E). We found that younger patients $(<40 \mathrm{y})$ had higher seropositivity for anti-S-RBDIgG $(P<0.05)$, and female patients exhibited increased seropositivity for NAbs $(P<0.05)$. Recent clinical trials have also reported a similar trend: younger individuals and female vaccine recipients exhibited stronger humoral immune responses to vaccination [2]. Interestingly, the patients undergoing nucleos(t)ide analog therapy had a significantly higher NAb titer than those who were not $(P<0.05)$ (Fig. 1D, E). Long-term antiviral therapy can inhibit viral replication and facilitate the restoration of the impaired immune system by recovering the function of circulating dendritic cells, natural killer cells, or T cells, particularly nucleotide analogs that can induce the production of IFN- $\lambda 3[6,8]$. These factors may account for the higher antibody titer in patients with antiviral therapy. Given that nucleos(t)ide analog therapy does not affect vaccine-induced immune responses, it should be continuously administered during vaccination to avoid negatively impacting CHB treatment.

\footnotetext{
${ }^{1}$ Department of Infectious Diseases, Union Hospital, Tongji Medical College, Huazhong University of Science and Technology, Wuhan, China. ${ }^{2}$ Joint International Laboratory of Infection and Immunity, Huazhong University of Science and Technology, Wuhan, China. ${ }^{3}$ These authors contributed equally: Tiandan Xiang, Boyun Liang, Hua Wang. email: bjwang73@163.com; xin11@hotmail.com
} 

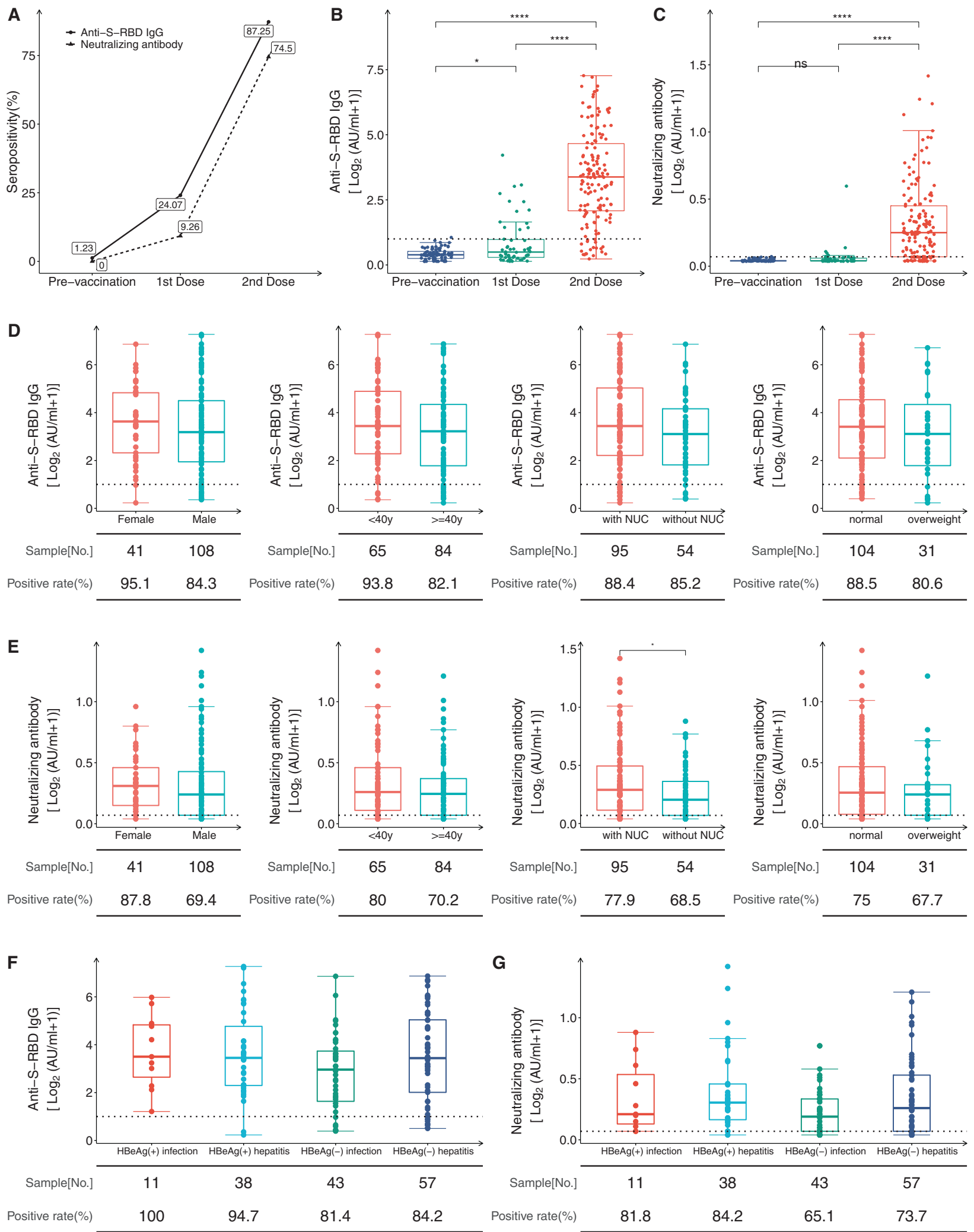

Fig. 1 Antibody responses following immunization with the inactivated vaccine in CHB patients. A The seropositivity of anti-S-RBD-lgG and NAbs in CHB patients. B, C Kinetics of the anti-S-RBD-IgG and NAb titers in vaccine-induced sera at different time points in CHB patients. Prevaccination, $n=81$; first dose, $n=54$; second dose, $n=149$. D, E The comparison of anti-S-RBD-IgG and NAb titers stratified according to sex, age, nucleos(t)ide analog (NUC) therapy, and BMI (overweight: BMI $\geq 25 ; 14$ patients had unavailable BMI values). F, G Comparison of antiS-RBD-lgG (F) and NAb titers (G) in $\mathrm{HBeAg}^{+}$chronic infection, $\mathrm{HBeAg}^{+}$chronic hepatitis, $\mathrm{HBeAg}^{-}$chronic infection, and $\mathrm{HBeAg}^{-}$chronic hepatitis individuals [9]. Sample numbers and positive rates are shown underneath. $P$ values were determined using a Mann-Whitney $U$ test or a Kruskal-Wallis test followed by Dunn's multiple comparisons test for antibody titers and Fisher's exact test for seropositivity. The horizontal dotted line represents the cutoff value. ns: no significance, ${ }^{*} p<0.05,{ }^{* * * *} p<0.0001$ 
Finally, we compared the antibody responses among the $\mathrm{CHB}$ patients in the various clinical stages of infection. The CHB participants were divided into four groups according to the "EASL 2017 Clinical Practice Guidelines on the Management of Hepatitis B Virus Infection" [9]: (I) HBeAg-positive chronic HBV infection, (II) HBeAg-positive chronic hepatitis B, (III) HBeAg-negative chronic HBV infection, and (IV) HBeAg-negative chronic hepatitis B. There was no significant difference in seropositivity or antibody titers among the four groups constituting the 149 CHB patients (Fig. 1F, $\mathrm{G})$, suggesting the general applicability of the inactivated vaccines within this patient population.

Altogether, our study reveals that SARS-CoV-2 inactivated vaccines achieve a favorable safety profile and efficient immunogenicity in patients with $\mathrm{CHB}$ in real-world vaccination scenarios. The results are encouraging despite some patients not being vaccinated following the standard dose interval time in clinical trials or the two dosages of the inactivated vaccine not being from the same manufacturer.

\section{REFERENCES}

1. Polack FP, Thomas SJ, Kitchin N, Absalon J, Gurtman A, Lockhart S, et al. Safety and efficacy of the BNT162b2 mRNA Covid-19 vaccine. N Engl J Med. 2020;383:2603-15.

2. Tanriover MD, Doganay HL, Akova M, Guner HR, Azap A, Akhan S, et al. Efficacy and safety of an inactivated whole-virion SARS-CoV-2 vaccine (CoronaVac): interim results of a double-blind, randomised, placebo-controlled, phase 3 trial in Turkey. Lancet. 2021;398:213-22.

3. Cornberg M, Buti M, Eberhardt CS, Grossi PA, Shouval D. EASL position paper on the use of COVID-19 vaccines in patients with chronic liver diseases, hepatobiliary cancer and liver transplant recipients. J Hepatol. 2021;74:944-51.

4. Wang J, Hou Z, Liu J, Gu Y, Wu Y, Chen Z, et al. Safety and immunogenicity of COVID-19 vaccination in patients with non-alcoholic fatty liver disease (CHESS2101): a multicenter study. J Hepatol. 2021;75: 439-41.

5. Thuluvath PJ, Robarts $P$, Chauhan M. Analysis of antibody responses after COVID19 vaccination in liver transplant recipients and those with chronic liver diseases. $J$ Hepatol. 2021; Online ahead of print.

6. Liu N, Liu B, Zhang L, Li H, Chen Z, Luo A, et al. Recovery of circulating CD56(dim) NK cells and the balance of Th17/Treg after nucleoside analog therapy in patients with chronic hepatitis B and low levels of HBsAg. Int Immunopharmacol. 2018;62:59-66.

7. Moon AM, Webb GJ, Aloman C, Armstrong MJ, Cargill T, Dhanasekaran R, et al. High mortality rates for SARS-CoV-2 infection in patients with pre-existing chronic liver disease and cirrhosis: Preliminary results from an international registry. J Hepatol. 2020;73:705-8.

8. Murata K, Asano M, Matsumoto A, Sugiyama M, Nishida N, Tanaka E, et al. Induction of IFN-lambda3 as an additional effect of nucleotide, not nucleoside, analogues: a new potential target for HBV infection. Gut. 2018;67:362-71.

9. EASL. 2017 Clinical Practice Guidelines on the management of hepatitis B virus infection. J Hepatol. 2017;67:370-98.

\section{ACKNOWLEDGEMENTS}

This study was supported by the Applied Basic and Frontier Technology Research Project of Wuhan (2020020601012233); the Science and Technology Key Project on Novel Coronavirus Pneumonia, Hubei Province (2020FCA002); the Fundamental Research Funds for the Central Universities (2020kfyXGYJ016 and 2020kfyXGYJ028); the National Science and Technology Major Project of China (2018ZX10302206, 2018ZX10723203, and 2017ZX10304402-002-005); the National Key R\&D Program of China (2017YFC0908104); and the Innovation Team Project of the Health Commission of Hubei Province (WJ2019C003). The authors thank AiMi Academic Services and Diana Liu for English language editing. We wish to thank the assistance given by Jia Liu, Cheng Peng, Jun $\mathrm{Wu}$, Juan Xu, Xuemei Feng, and Rong Zhang in patients enrollment and sample collection.

\section{AUTHOR CONTRIBUTIONS}

$X Z$, BJW, TDX, and BYL designed and conceived the study; TDX, BYL, and HW performed the experiments; TDX, BYL, HW, XFQ, HLZ, YWH, DLY, BJW, and XZ enrolled patients and acquired the data; $\mathrm{BYL}$ and $\mathrm{HW}$ analyzed the data and contributed to producing the charts; TDX drafted the manuscript; XZ and BJW revised the manuscript.

\section{COMPETING INTERESTS}

The authors declare no competing interests.

\section{ADDITIONAL INFORMATION}

Supplementary information The online version contains supplementary material available at https://doi.org/10.1038/s41423-021-00795-5.

Correspondence and requests for materials should be addressed to Baoju Wang or Xin Zheng.

Reprints and permission information is available at http://www.nature.com/ reprints

\begin{abstract}
Open Access This article is licensed under a Creative Commons Attribution 4.0 International License, which permits use, sharing, adaptation, distribution and reproduction in any medium or format, as long as you give appropriate credit to the original author(s) and the source, provide a link to the Creative Commons license, and indicate if changes were made. The images or other third party material in this article are included in the article's Creative Commons license, unless indicated otherwise in a credit line to the material. If material is not included in the article's Creative Commons license and your intended use is not permitted by statutory regulation or exceeds the permitted use, you will need to obtain permission directly from the copyright holder. To view a copy of this license, visit http://creativecommons. org/licenses/by/4.0/.
\end{abstract}

(c) The Author(s) 2021 